\title{
BMJ Open Dementia among migrants and ethnic minorities in Italy: rationale and study protocol of the ImmiDem project
}

\author{
Marco Canevelli (D) , ${ }^{1,2}$ Eleonora Lacorte, ${ }^{1}$ Ilaria Cova, ${ }^{3}$ Silvia Cascini, ${ }^{4}$ \\ Anna Maria Bargagli, ${ }^{4}$ Laura Angelici, ${ }^{4}$ Angela Giusti (D) , ${ }^{1}$ Simone Pomati, ${ }^{3}$ \\ Leonardo Pantoni, ${ }^{5}$ Nicola Vanacore, ${ }^{1}$ On behalf of the ImmiDem Study Group
}

To cite: Canevelli M, Lacorte E, Cova I, et al. Dementia among migrants and ethnic minorities in Italy: rationale and study protocol of the ImmiDem project. BMJ Open 2020;10:e032765. doi:10.1136/ bmjopen-2019-032765

- Prepublication history and additional material for this paper are available online. To view these files, please visit the journal online (http://dx.doi. org/10.1136/bmjopen-2019032765).

Received 04 July 2019 Revised 03 October 2019 Accepted 13 November 2019

Check for updates

(c) Author(s) (or their employer(s)) 2020. Re-use permitted under CC BY-NC. No commercial re-use. See rights and permissions. Published by BMJ.

${ }^{1}$ National Center for Disease

Prevention and Health

Promotion, National Institute of Health, Rome, Italy

${ }^{2}$ Department of Human

Neuroscience, Sapienza University, Rome, Italy

${ }^{3}$ Center for Research and

Treatment on Cognitive

Dysfunctions, Luigi Sacco

University Hospital, Milan, Italy

${ }^{4}$ Department of Epidemiology,

Regional Health Service, Lazio

Region, Rome, Italy

${ }^{5}$ Department of Biomedical and

Clinical Sciences Luigi Sacco,

University of Milan, Milan, Italy

Correspondence to

Dr Marco Canevelli;

marco.canevelli@gmail.com

\section{ABSTRACT}

Introduction Due to the ongoing demographic and epidemiological transitions, estimating the phenomenon of dementia in migrants and minority groups, exploring its characteristics and challenges and implementing dedicated healthcare policies, constitute emerging and urgent matters for Western countries. In the present paper we describe the rationale and design of the 'Dementia in immigrants and ethnic minorities living in Italy: clinicalepidemiological aspects and public health perspectives" (ImmiDem) project.

Methods and analysis Three main aims will be pursued by the ImmiDem project. First, a survey of all Italian dementia services will be conducted with dedicated questionnaires in order to estimate and describe the proportion and characteristics of migrants seeking help for cognitive disturbances. The different clinical approaches for diagnosing dementia and the challenges encountered in the assessment of cognitive functioning and in the provision of care in these groups of individuals will also be investigated. Second, record linkage procedures of data routinely collected in regional Health Information Systems will be conducted in order to identify and monitor migrant individuals with dementia living in the Lazio region. Third, tailored national and local care-coordination pathways and/or good practices dedicated to migrants affected by dementia and cognitive disorders will be identified and promoted.

Ethics and dissemination The study protocol was approved by the Ethics Committee of the Italian National Institute of Health (protocol 10749; 5 April 2018). The project was launched in November 2018 and will end in November 2021. The findings of the project will be disseminated through scientific peer-reviewed journals as well as to the public via the Dementia Observatory website (https://demenze.iss.it).

\section{INTRODUCTION}

\section{Background}

The intersection between ageing and migration represents an emerging issue for Western countries and their healthcare systems. ${ }^{1}$ In fact, a steadily increase in longevity is observed not only among native individuals but also among migrants, conceived as ' ... any person who is moving or has moved across an

\section{Strengths and limitations of this study}

- The ImmiDem project represents the first initiative aimed at characterising the issue of dementia among migrants and ethnic minorities in Italy.

- The project will contribute with unique data on the dimensions and characteristics of this emerging phenomenon, documenting possible inequalities across Italian regions and services.

- The methodological design, consisting of both quantitative and qualitative research approaches, will allow to explore this issue multidimensionally and from a 'real-world' perspective.

- The low adhesion of dementia services to the survey might represent a potential risk for the project.

- The issue of dementia in migrants might be underrepresented at the level of Italian dementia services and, thus, only partially captured by the ImmiDem project.

international border or within a State away from his/her habitual place of residence, regardless of the person's legal status, whether the movement is voluntary or involuntary, what the causes for the movement are or what the length of the stay is'. ${ }^{2}$ Accordingly, the proportion of older international migrants is rapidly growing in most of countries. For instance, the number of $>60$-year-old foreignborn individuals living in Europe has nearly doubled in the last three decades, growing from 8876791 in 1990 to 15840486 in $2017 .^{3}$ Therefore, it can be expected that older migrants, including those who have recently arrived and those who are ageing in their country of destination (ageing in place), will increasingly deal with the burden of agerelated chronic diseases. ${ }^{1}$ Migration, due to its multidimensional nature, adds a further layer of complexity to the already great diversity (in terms of phenotypic expression, trajectories and management) of these pathological conditions. Consequently, diversity-sensitive healthcare practices and policies are required 
to encounter the clinical and social needs of affected individuals with a migration background. ${ }^{14}$

More specifically, the occurrence of dementia and cognitive disorders among migrants will likely assume (and is probably already assuming) a special relevance for several reasons. ${ }^{5-7}$ First, the detection of dementia in these individuals is frequently hampered by the linguistic barrier, the reduced literacy/educational level and the scarce availability and diffusion of cross-culturally valid instruments supporting a reliable cognitive testing. In parallel, diverse sociocultural determinants and healthcare inadequacies may postpone help-seeking for cognitive disturbances. As a potential consequence, migrants have a lower likelihood of receiving a dementia diagnosis and treatment compared with native individuals, as suggested by various studies conducted in European countries. ${ }^{89}$ Moreover, once the diagnosis of dementia is established, patients with a migration background frequently experience a reduced support and care and a difficult access to healthcare services. ${ }^{10}$ Individuals from different cultures may have different beliefs, perceptions and understandings towards dementia and cognitive disorders with important implications in terms of use of resources. Finally, most dementia facilities have limited experience with migrant patients and with culture-sensitive models of care provision, and consider (by their own admission) the approach to and management of cognitive disturbances in these individuals to be challenging. ${ }^{7}$ It is noteworthy that this issue has already reached relevant dimensions considering that nearly 475000 dementia cases can be estimated among migrants living in Europe. ${ }^{11}$ All these considerations may likely assume further relevance in those migrants composing ethnic minorities, defined as any '...group which is numerically inferior to the rest of the population of a State and in a non-dominant position, whose members possess ethnic, religious or linguistic characteristics which differ from those of the rest of the population and who, if only implicitly, maintain a sense of solidarity directed towards preserving their culture, traditions, religion or language'. ${ }^{12}$

Based on these considerations, estimating the phenomenon of dementia in migrants and minority groups, investigating its characteristics and challenges and implementing dedicated healthcare policies, constitute emerging and urgent matters for Western countries. This has recently been emphasised in the Global Action Plan on dementia promulgated by the WHO which stresses how the public health responses to this condition must be taken in a diversity-sensitive perspective, that is considering the needs of vulnerable people including migrants. ${ }^{13}$

The aim of the present article is to describe the rationale and design of the 'Dementia in immigrants and ethnic minorities living in Italy: clinical-epidemiological aspects and public health perspectives' (ImmiDem) project. This initiative aims at exploring, for the first time, the public health relevance of dementia in the migrant population living in Italy.

\section{Hypotheses and objectives}

In this section, the hypotheses that have driven the rationale and conception of ImmiDem and the pertaining objectives that will be pursued by the project are described.

Hypothesis 1 Population ageing is leading to a marked increase in dementia prevalence among migrants and ethnic minorities living in Western countries. Therefore, it might be expected that more migrant individuals will seek help for cognitive disturbances and will attend dementia services in Italy, with important public health and societal implications. In the absence of specific recommendations, it is likely that substantial discrepancies exist across services in the clinical approach to dementia in such populations. Surveying the (probably) different practices and the main challenges encountered in the provision of care is the first step for identifying potentially standardised and common strategies.

Aim 1. To estimate and describe the proportion and characteristics of migrants seeking help for cognitive disturbances and/or attending dementia services in Italy. To systematically survey the different clinical approaches and tools adopted for diagnosing dementia among migrants and minority groups across Italian Centers for Cognitive Disorders and Dementia (CCDDs), with special attention to cognitive/neuropsychological testing, and the practical challenges encountered in the assessment of cognitive functioning, identification of dementia and post-diagnostic care and support in these specific populations of individuals.

Hypothesis 2. Demographic and clinical data routinely collected in national and regional Health Information Systems (HIS) could be used to identify and monitor, through record linkage procedures, individuals with a migration background affected by dementia.

Aim 2. To identify and monitor over time migrants individuals with dementia living in an Italian region. Lazio will be taken as example to characterise the phenomenon at the regional level.

Hypothesis 3. The design and implementation of tailored care-coordination pathways and good practices may contribute to enhance the quality of care of immigrants with dementia and optimise the allocation of healthcare resources dedicated to such rising issues.

Aim 3. To identify and promote tailored national and locally oriented care-coordination pathways or good practices dedicated to migrants affected by dementia and cognitive disorders.

\section{The Italian scenario}

Italy is one of the main destination of migratory flows in Europe, with nearly 6 million foreign residents currently living in the country. The number of people with a migration background has steadily increased over the last decades, passing from 1428221 in 1990 to 5907461 in 2017. Moreover, the migrant population residing in Italy is progressively getting older. In fact, the number of $>65$-year-old migrants living in the country has gradually 


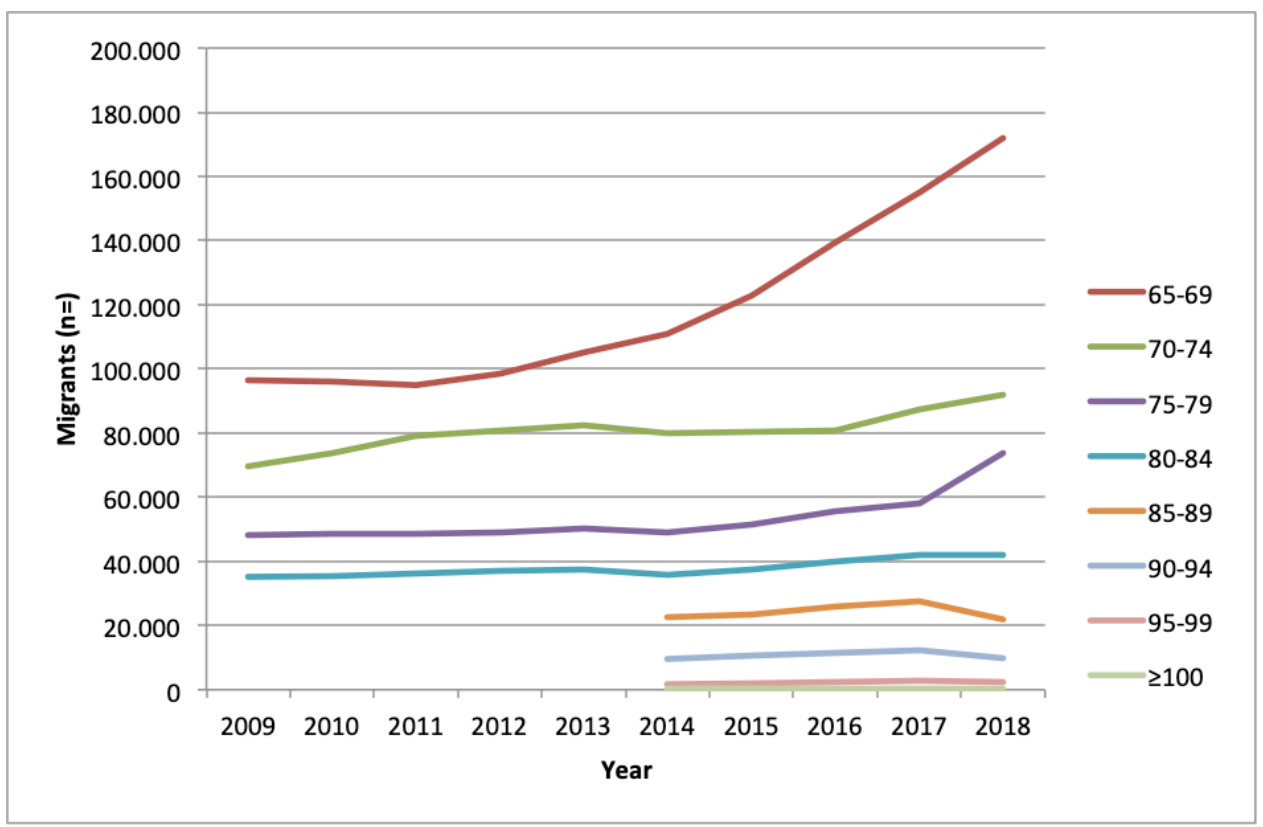

Figure 1 Number of older migrants living in Italy in the 2009 to 2018 period per age classes. Source: Eurostat $2018{ }^{14}$

increased from 277661 in 2009 to 414384 in 2018 (ie, a $67 \%$ increase) (figure 1$).{ }^{14}$

In parallel, more than one million individuals with dementia live in Italy, with numbers expected to markedly increase in the near future. ${ }^{15}$ To date, nearly 2000 services, including memory clinics (named CCDDs), daycare services and residential care facilities, are devoted to the management of patients with dementia. The first Italian Dementia National Plan was defined in 2014 in order to improve and harmonise the process of taking care of people with dementia. ${ }^{15}$

Lazio is the third most populated region of Italy, with 5896693 residents registered at the beginning of 2018 . $^{16}$ It covers an area of $17236 \mathrm{~km}^{2}$ and includes five provinces and 378 municipalities. It is divided into 12 local health units and 56 health districts. A total of 24512 foreignborn individuals lived in the region in 2017. Nearly 90000 cases of dementia among $>65$-year-old individuals can be estimated among its residents. ${ }^{17}$

The magnitude of the issue of dementia among migrants in Italy has recently been estimated by applying dementia prevalence rates to the migrant population residing in the country. Nearly 7700 dementia cases were estimated in these individuals (5022 among women, 2725 among men), with a marked inter-regional variability. ${ }^{18}$ Among them, 1065 cases were calculated in the Lazio region.

\section{METHODS AND ANALYSIS}

\section{Definition of migrant and ethnic minority}

There is no universally accepted definition for migrant at an international level and in the scientific community. ${ }^{19}$ In the present project, the definition proposed by the International Organization for Migration (IOM) will be adopted: “...any person who is moving or has moved across an international border or within a State away from his/her habitual place of residence, regardless of the person's legal status, whether the movement is voluntary or involuntary, what the causes for the movement are or what the length of the stay is'. ${ }^{2}$ In order to capture the heterogeneous health needs of migrants, all the activities and analyses will account for the attributes of the diverse migrant groups (with regard to their legal status, reason for migration, country of origin, ethnicity, socioeconomic status).$^{20}$

Despite the lack of a universally accepted definition in international law, a minority can be conceived as '...a group which is numerically inferior to the rest of the population of a State and in a non-dominant position, whose members possess ethnic, religious or linguistic characteristics which differ from those of the rest of the population and who, if only implicitly, maintain a sense of solidarity directed towards preserving their culture, traditions, religion or language'. 12

\section{Aim 1 - survey of Italian dementia services}

The public health relevance of dementia among migrants living in Italy will be explored through the 'Dementia Observatory'. This website consists in an online map of all the Italian services (ie, CCDDs, daycare services and residential care facilities) dedicated to dementias. ${ }^{21}$ The main information (eg, address, contacts, opening hours, staff composition, how to access the service) on each of these facilities is available on the web-platform and is constantly updated. This activity is part of the 'Survey of the social and health services dedicated to dementias and creation of a specific website called Observatory for dementias' project that was assigned to the National Center of Epidemiology and Health Promotion of the National Institute of Health in the context of the 2013 central actions of the National Center for Disease Prevention and Control of the Ministry of Health. ${ }^{22}$ A total of 1860 health and 

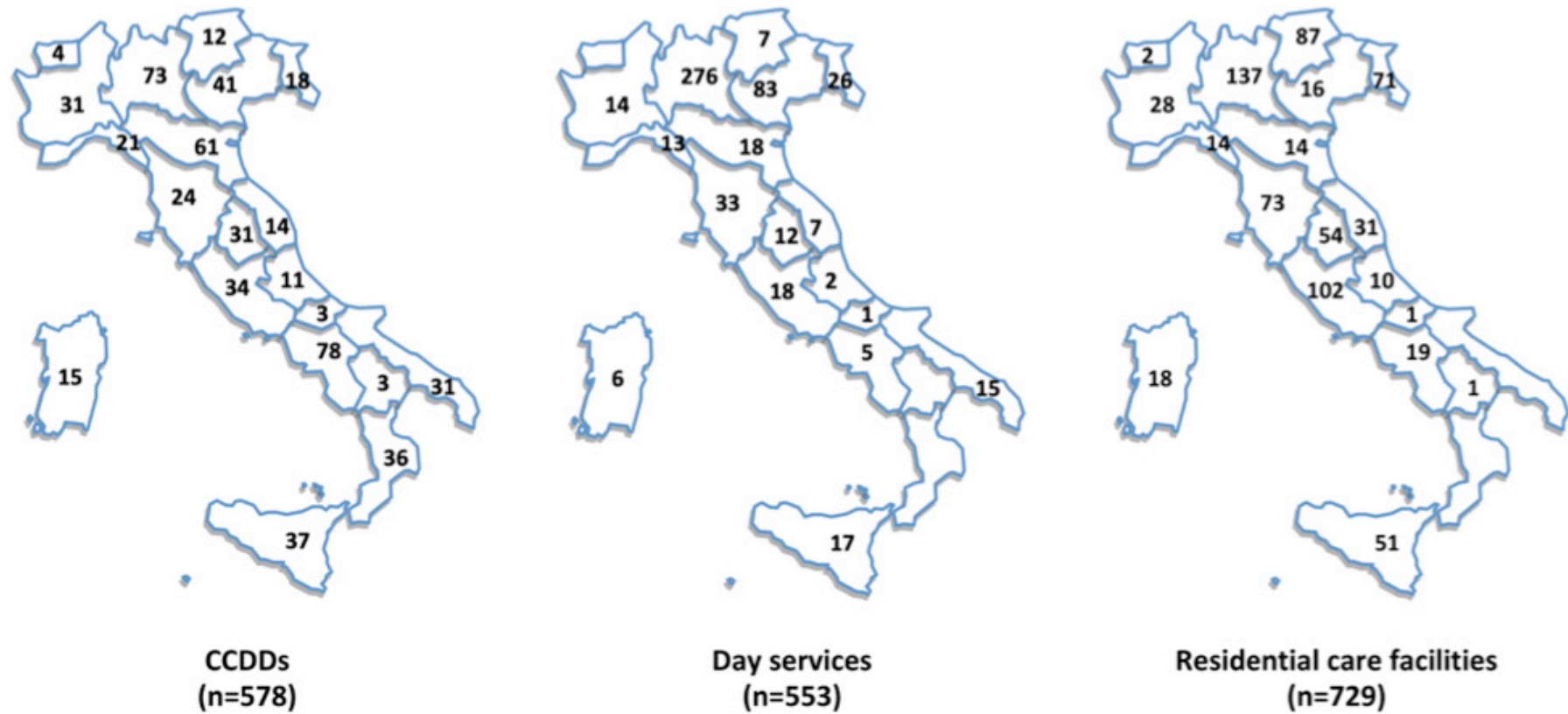

Figure 2 Currently mapped Italian dementia services (updated to June 2019). CCDDs, Centers for Cognitive Disorders and Dementia. Source: Italian Dementia Observatory. ${ }^{21}$

social health structures, totally or partially covered by the National Health Service, are included in the website (update to June 2019) (figure 2).

A questionnaire will be designed and developed to properly capture the proportion and the main sociodemographic and clinical characteristics of migrants and individuals composing ethnic minority groups attending dementia services in Italy. A section of the questionnaire will specifically explore various aspects of the assessment of dementia in this special population (eg, adoption of translated questionnaires or cross-cultural screening/ assessment tools, involvement of additional professionals such as interpreters and/or cultural mediators). Finally, a set of questions will allow to identify the factors that are supposed to be the main barriers in the clinical approach and provision of care (eg, in terms of communication of diagnosis, assessment of neuropsychiatric symptoms, integration of care, compliance to treatments) dedicated to migrants with cognitive disorders according to the experience of Italian services (box 1).

During the exploratory phase of the study, individuals with a migration background presenting cognitive disturbances and their family caregivers will be involved in focus groups or in-depth interviews together with physicians, nurses, cultural mediators and social workers, in order to elaborate and calibrate the questionnaire addressed to Italian CCDDs. Focus groups will involve homogeneous social groups (eg, caregivers or health professionals). In parallel, individual interviews will be used to seek in-depth perspectives (eg, from individuals with different timing of diagnosis or different migration experiences). Participants will be enrolled using a convenience sampling methodology. Only patients with mild-to-moderate cognitive deficits, able to provide their informed consent, will be considered for participation in such activities. Focus groups and in-depth interviews will be conducted, in different languages, by expert facilitators using a semistructured guide. Interpreters and cultural mediators will be involved if necessary. A thematic analysis will be performed, based on both inductive and deductive approach. The NVivo software for qualitative data analysis will be used for data coding and classification. This qualitative approach ${ }^{23}$ will contribute to explore the issue of dementia among migrants also considering the attitudes, beliefs and perceptions on dementia of individuals from diverse cultural groups. Moreover, the experiences of different professionals already in contact with this phenomenon will be valued. This should ultimately allow to develop targeted and feasible healthcare pathways (Aim 3).

The developed questionnaire will be validated in a pilot phase involving a convenience sample of dementia services (CCDDs, daycare services and residential care facilities). A tutorial activity will be also available for all the participating centres. ${ }^{24}$ The instrument will be then submitted to all the services mapped in the 'Dementia Observatory'. Additional multidisciplinary focus groups will be conducted in order to interpret and discuss the findings of both the pilot phase and the whole survey. A total of three to four focus groups will be conducted during the project.

\section{Aim 2 - identification of migrants with dementia living in the Lazio region}

Migrants affected by dementia residing in the Lazio region will be identified through record linkage procedures, using an algorithm based on data from HIS already tested at the national and international level. ${ }^{25-27}$ All residents of Lazio region served by the Regional Health Service are uniquely assigned a personal identification number 
Box 1 Main contents of the survey addressed to Italian dementia services (Aim 1)

\section{Section 1. Characteristics of the service}

Type of service (eg, Centers for Cognitive Disorders and Dementia vs day service vs residential).

Affiliation (eg, hospital vs university vs community).

Professionals (eg, neurologists, geriatricians, psychiatrists, neuropsychologists).

Activities (eg, clinical assessment, neuropsychological testing, management of pharmacological therapies, non-pharmacological interventions, counselling).

How to access the service.

\section{Section 2. Data of the service}

Number of referrals and assessments (natives vs migrants).

Number of dementia diagnosis (natives vs migrants).

Proportion of referral sources (natives vs migrants).

\section{Section 3. Resources for migrants with dementia/cognitive} disturbances

Personnel with knowledge of a second language.

Personnel with a migration background.

Interpreter/social worker.

Availability of translated information material.

Contacts with social services, interpreters, associations for migrants/ ethnic minorities, religious figures.

Section 4. Cognitive assessment of migrants with dementia/cognitive disturbances

Availability of translated screening/assessment tools.

Use of cross-cultural screening/assessment tests.

Adoption of dedicated test batteries.

Section 5. Complexities in the approach to dementia/ cognitive disturbances among migrants

Factors complicating/hindering the diagnostic approach.

Main difficulties in the management of dementia.

Competencies/preparedness of the service. recorded in all the regional healthcare databases. This individual identifier makes it possible to link different databases and to identify individuals avoiding duplicates.

The following databases will be used: (a) Hospital Discharge Registry, (b) Drug Prescription Registry, (c) Ticket Exemption Registry, (d) Regional Health Assistance File and (e) Mortality Registry (table 1). Dementia cases will be ascertained applying the following algorithm: 1. At least one hospitalisation with a primary or a secondary diagnosis of dementia (International Classification of Diseases, Ninth Revision, Clinical Modification diagnostic code 290.XX, 291.2, 292.82, 294.1, 046.1, 331.0, 331.1, 331.2, 331.82, 331.89, 331.9 (see online supplementary material) or ICD-10 code F00-F03, G30 after 2002) $)^{28}$, OR

2. At least one prescription of any anti-dementia drug (Anatomical Therapeutic Chemical codes N06D), OR

3. A co-payment exemption claim specific for dementia (codes 011 and 029).

During the project, the possibility to use data from other HIS for case ascertainment will also be evaluated (eg, specialist outpatient services, residential long-term care and home care facilities, rehabilitation services).

The definition of the immigrant status will be based on the country of birth as registered in the Regional Health Assistance File. Another source of data will be the Municipal Population Registry of Rome that collects information on citizenship for all the city residents.

\section{Aim 3 - identification and promotion of care-coordination pathways and good practices}

The procedures pertaining to Aim 1 and Aim 2 will allow to better characterise the issue of dementia among migrants and ethnic minorities in Italy under a clinical and public health perspective. The adopted methodologies will also allow the accurate tracking of the pathways of migrant

Table 1 Health Information Systems databases used for the identification of dementia cases in the Lazio region (Aim 2)

\begin{tabular}{|c|c|}
\hline Database & Description \\
\hline Hospital discharge registry & $\begin{array}{l}\text { Data from all regional hospitals, including individual information on patients' } \\
\text { characteristics, admission and discharge dates, discharge diagnoses according to the } \\
\text { ICD-9-CM. }\end{array}$ \\
\hline PHARM & $\begin{array}{l}\text { Individual records for each drug prescription dispensed in public and private pharmacies } \\
\text { belonging to the regional health authorities and referring to residents. The registry is limited } \\
\text { to drugs that are reimbursed by the healthcare system referring to outpatients. Drugs } \\
\text { are identified by the national drug register code, which refers to the international ATC } \\
\text { classification system. }\end{array}$ \\
\hline PHARMED & $\begin{array}{l}\text { Individual records for each drug prescription dispensed by hospital at discharge and by } \\
\text { specialist outpatient clinics. The database includes the information also gathered by the } \\
\text { PHARM registry. }\end{array}$ \\
\hline Ticket exemption registry & $\begin{array}{l}\text { Data on all residents who are entitled to co-pay fee exemptions for some particular } \\
\text { conditions (eg, disability, chronic diseases, low income or old age). }\end{array}$ \\
\hline Regional health assistance file & Records of all resident individuals registered within the regional health service. \\
\hline Regional mortality registry & $\begin{array}{l}\text { Data on patients' demographic characteristics, as well as on the date, place and cause of } \\
\text { death (codified by ICD-9 codes). }\end{array}$ \\
\hline
\end{tabular}

ATC, Anatomical Therapeutic Chemical; ICD-9-CM, International Classification of Diseases, Ninth Revision, Clinical Modification. 
Table 2 Timeline of the ImmiDem project

Months

$\begin{array}{lllllllllllllllllll}2 & 4 & 6 & 8 & 10 & 12 & 14 & 16 & 18 & 20 & 22 & 24 & 26 & 28 & 30 & 32 & 34 & 36\end{array}$

Aim 1

Questionnaire set-up

Questionnaire pilot phase

Focus groups on the results of the pilot phase

Questionnaire administration to all Italian dementia services

Focus groups on the results of the whole survey

Aim 2

Record linkage procedures in the Lazio region

Aim 1 and 2

Analysis of collected data

Dissemination of results

Aim 3

Elaboration of a model of care-coordination pathway

Aim 1, 2 and 3

Kick-off meeting

Final conference

patients with dementia within the healthcare system, thus tentatively identifying the main obstacles and barriers. Altogether, these emerging findings should support the elaboration of a policy document about how to implement specific care-coordination pathways on this issue. Further references for the preparation and calibration of this instrument will be obtained by conducting dedicated systematic reviews of both the scientific literature and national/international healthcare policies aimed at identifying effective models of care and good/best practices. Moreover, some of the ongoing care-coordination pathways that are already ongoing in Italy (ie, in the Lombardia region and in the city of Rome) will be analysed to retrieve specific information on how migrants with dementia are already using healthcare resources. The final document will ultimately be shared with and disseminated to the diverse stakeholders (eg, institutions, policymakers, scientific societies, general practitioners, associations of patients) involved in the management of dementia and cognitive disorders.

\section{Duration}

The ImmiDem project will have a duration of 36 months. The timeline of the project is illustrated in table 2. The project was launched in November 2018 and will end in November 2021.

The collection of data from Italian dementia services (Aim 1) started at the end of July 2019 and will tentatively be completed by April 2020. The record linkage procedures of data coming from Health Information Systems in the Lazio region (Aim 2) have already been launched in May 2019 and will be completed by April 2020.

\section{Patient and public involvement}

Individuals with a migration background presenting cognitive disturbances and their family caregivers will be involved in focus groups or in-depth interviews together with physicians, nurses, cultural mediators and social workers in order to elaborate and calibrate the questionnaire addressed to Italian CCDDs (Aim 1). This approach will contribute to explore the issue of dementia among migrants also considering the attitudes, beliefs and perceptions on dementia of individuals from diverse cultural groups. Moreover, the experiences of different professionals already in contact with this phenomenon will be valued. This should ultimately allow to develop targeted and feasible healthcare pathways (Aim 3).

All the data and findings of the project will be shared with the public on the Dementia Observatory website (https://demenze.iss.it).

\section{Ethics and dissemination}

The principles of the Declaration of Helsinki have been followed to design and develop the ImmiDem project.

The Department of Epidemiology of Lazio Regional Health Service is the regional centre of referral for epidemiological research and has full access to anonymised health information systems. Consent collection within the procedures of Aim 2 (ie, identification of migrants with dementia living in the Lazio region) is not necessary because data of interest are already collected in the regional HIS and will be analysed anonymously through a standardised methodology in compliance with the national privacy law (national legislative decree on privacy policy no. 196/30 June 2003). Therefore, individuals 
cannot be identified directly or through identifiers. Moreover, results will be shown in aggregate form.

The findings of the project will be disseminated through scientific peer-reviewed journals as well as to the public via the Dementia Observatory website (https:// demenze.iss.it).

\section{DISCUSSION}

To the best of our knowledge, ImmiDem constitutes the first initiative aimed at characterising the issue of dementia among migrants and ethnic minorities in Italy with a clinical-epidemiological and public health perspective. The project will contribute with unique data concerning the dimensions and features of this emerging phenomenon in Italy, documenting possible inequalities across regions and services and capturing the various strategies and instruments currently adopted for approaching it in the clinical practice as well as the challenges encountered. This information will allow to tentatively identify dedicated care-coordination pathways and good practices aimed at improving the standards of care and minimising its social and healthcare burdens.

The adopted methodologies (ie, survey of dementia services and record linkage procedures within regional HIS) will permit us to reflect this clinical and social challenge from a -real-world' viewpoint, thus increasing the practical implications of the findings and their transferability. Moreover, the issue will be addressed in a multidimensional way, giving relevance to its main facets and complexities (eg, cross-cultural cognitive testing, use of resources, competency of services). The adoption of a mixed-methods approach incorporating also qualitative research methods (ie, focus groups or in-depth interviews) will allow to properly calibrate the different activities based on the personal and/or professional experience of individuals who are exposed to the phenomenon on a daily basis. ${ }^{29}$ On the other hand, several potential limitations of the project should be acknowledged and discussed. A potential risk may be represented by the low adhesion to the survey directed to Italian dementia services. Nevertheless, it is noteworthy that the 'Dementia Observatory' has already been used to survey Italian dementia facilities with high response rates. ${ }^{22}{ }^{30}$ Moreover, in order to enhance participation, the project will be presented and disseminated in scientific and social manifestations. Second, exploratory data suggest that the issue of dementia among migrants is still under-represented in Italian dementia services. ${ }^{31}{ }^{32}$ It could be expected that only a limited proportion of individuals with a migration background and belonging to minority groups might seek help for cognitive disturbances and that diverse barriers and inadequacies, both at the service and community level, might limit their access to our memory clinics. Therefore, widening the focus to other settings (eg, primary care) would contribute to achieve a better comprehension of the phenomenon. Finally, ImmiDem should ideally be federated with similar initiatives conducted in other
European countries in order to address this issue from an extended perspective.

Collaborators ImmiDem Study Group: Ilaria Bacigalupo, Guido Bellomo, Giuseppe Gervasi, Francesca Marchetti, Flavia Mayer, Monica Mazzola, Ilaria Palazzesi, Paola Piscopo, Mariacristina Porrello, Emanuela Salvi, Valerio Zaccaria, Francesca Zambri (National Center for Disease Prevention and Health Promotion, National Institute of Health, Rome, Italy); Giuseppe Bruno, Martina Valletta (Department of Human Neuroscience, Sapienza University, Rome, Italy).

Contributors Study design: MC, EL, IC, SC, AMB, LA, AG, SP, LP, NV. Study conduction: MC, EL, IC, SC, AMB, LA, AG, SP, LP, NV. Writing the manuscript: MC, NV. Drafting the manuscript: EL, IC, SC, AMB, LA, AG, SP, LP.

Funding The "Dementia in immigrants and ethnic minorities living in Italy: clinical-epidemiological aspects and public health perspectives" (ImmiDem) project is supported by a research grant from the Italian Ministry of Health (GR-2016-02364975).

Map disclaimer The depiction of boundaries on this map does not imply the expression of any opinion whatsoever on the part of BMJ (or any member of its group) concerning the legal status of any country, territory, jurisdiction or area or of its authorities. This map is provided without any warranty of any kind, either express or implied.

Competing interests None declared.

Patient consent for publication Not required.

Ethics approval The Ethics Committee of the Italian National Institute of Health approved the study protocol (protocol 10749; 5 April 2018).

Provenance and peer review Not commissioned; externally peer reviewed.

Open access This is an open access article distributed in accordance with the Creative Commons Attribution Non Commercial (CC BY-NC 4.0) license, which permits others to distribute, remix, adapt, build upon this work non-commercially, and license their derivative works on different terms, provided the original work is properly cited, appropriate credit is given, any changes made indicated, and the use is non-commercial. See: http://creativecommons.org/licenses/by-nc/4.0/.

\section{ORCID iDs}

Marco Canevelli http://orcid.org/0000-0001-7333-6478

Angela Giusti http://orcid.org/0000-0001-8695-7847

\section{REFERENCES}

1 World Health Organization. Health of older refugees and migrants, 2018. Available: http://www.euro.who.int/en/publications/abstracts/ health-of-older-refugees-and-migrants-2018 [Accessed 15 Jan 2019].

2 International Organization for Migration. Who is a migrant? 2016. Available: https://www.iom.int/who-is-a-migrant

3 United Nations Population Division. Department of economic and social Affairs. Available: http://www.un.org/en/development/desa/ population/migration/data/estimates2/estimates17.shtml [Accessed 10 Jan 2019].

4 Abubakar I, Aldridge RW, Devakumar D, et al. The UCL-Lancet Commission on migration and health: the health of a world on the move. Lancet 2018;392:2606-54.

5 Cooper C, Tandy AR, Balamurali TBS, et al. A systematic review and meta-analysis of ethnic differences in use of dementia treatment, care, and research. Am J Geriatr Psychiatry 2010;18:193-203.

6 Kenning C, Daker-White G, Blakemore A, et al. Barriers and facilitators in accessing dementia care by ethnic minority groups: a meta-synthesis of qualitative studies. BMC Psychiatry 2017;17:316.

7 Nielsen TR, Vogel A, Riepe MW, et al. Assessment of dementia in ethnic minority patients in Europe: a European Alzheimer's disease Consortium survey. Int Psychogeriatr 2011;23:86-95.

8 Diaz E, Kumar BN, Engedal K. Immigrant patients with dementia and memory impairment in primary health care in Norway: a national registry study. Dement Geriatr Cogn Disord 2015;39:321-31.

9 Stevnsborg L, Jensen-Dahm C, Nielsen TR, et al. Inequalities in access to treatment and care for patients with dementia and immigrant background: a Danish nationwide study. J Alzheimers Dis 2016;54:505-14.

10 Mukadam N, Cooper C, Livingston G. A systematic review of ethnicity and pathways to care in dementia. Int $J$ Geriatr Psychiatry 2011;26:12-20. 
11 Canevelli M, Lacorte E, Cova I, et al. Estimating dementia cases amongst migrants living in Europe. Eur J Neurol 2019;26:1191-9.

12 International Organization for Migration. Glossary on migration. 2nd edn, 2011.

13 WHO. Global action plan on the public health response to dementia 2017 - 2025. WHO. Available: http://www.who.int/mental_health/ neurology/dementia/action_plan_2017_2025/en/ [Accessed 15 Jan 2019].

14 Database - Eurostat. Available: https://ec.europa.eu/eurostat/web/ population-demography-migration-projections/data/database [Accessed 10 Jan 2019].

15 Di Fiandra T, Canevelli M, Di Pucchio A, et al. The Italian dementia national plan. Commentary. Ann Ist Super Sanita 2015;51:261-4.

16 Istat.it Popolazione E famiglie. Available: https://www.istat.it/it/ popolazione-e-famiglie?dati [Accessed 14 Jan 2019].

17 Bacigalupo I, Mayer F, Lacorte E, et al. A systematic review and meta-analysis on the prevalence of dementia in Europe: estimates from the Highest-Quality studies adopting the DSM IV diagnostic criteria. J. Alzheimers Dis 2018;66:1471-81.

18 Canevelli M, Lacorte E, Cova I, et al. Estimating dementia cases in the immigrant population living in Italy. Neurol Sci 2018;39:1775-8.

19 How do variations in definitions of "migrant" and their application influence the access of migrants to health care services? (2016), 2018. Available: http://www.euro.who.int/en/publications/abstracts/ how-do-variations-in-definitions-of-migrant-and-their-applicationinfluence-the-access-of-migrants-to-health-care-services-2016 [Accessed 17 Jan 2019].

20 Rechel B, Mladovsky P, Ingleby D, et al. Migration and health in an increasingly diverse Europe. Lancet 2013;381:1235-45.

21 Mappa online dei servizi - Osservatorio demenze. Available: https:// demenze.iss.it/mappaservizi/ [Accessed 10 Jan 2019].
22 Di Pucchio A, Di Fiandra T, Marzolini F, et al. Survey of health and social-health services for people with dementia: methodology of the Italian national project. Ann Ist Super Sanita 2017;53:246-52.

23 Pope C. Qualitative methods in research on healthcare quality. Qual Saf Health Care 2002;11:148-52.

24 Boynton PM. Administering, analysing, and reporting your questionnaire. BMJ 2004;328:1372-5.

25 Dipartimento di Epidemiologia del Servizio Sanitario Regionale, Regione Lazio. Identificazione di popolazioni affette dA patologie $E$ stime di occorrenza. Edizione 2018.

26 Bharmal MF, Weiner M, Sands LP, et al. Impact of patient selection criteria on prevalence estimates and prevalence of diagnosed dementia in a Medicaid population. Alzheimer Dis Assoc Disord 2007;21:92-100.

27 Francesconi $\mathrm{P}$, Gini R, Roti L, et al. The Tuscany experimental registry for Alzheimer's disease and other dementias: how many demented people does it capture? Aging Clin Exp Res 2007;19:390-3.

28 Chen $\mathrm{H}$, Kwong JC, Copes R, et al. Exposure to ambient air pollution and the incidence of dementia: a population-based cohort study. Environ Int 2017;108:271-7.

29 Qualitative Research in European Migration Studies. Ricard ZapataBarrero. Springer. Available: https://www.springer.com/gp/book/ 9783319768601 [Accessed 13 Jun 2019].

30 Di Pucchio A, Vanacore N, Marzolini F, et al. Use of neuropsychological tests for the diagnosis of dementia: a survey of Italian memory clinics. BMJ Open 2018;8:e017847.

31 Canevelli M, Zaccaria V, Ruocco C, et al. Migrants seeking help for cognitive disturbances: exploratory data from an Italian memory clinic. Neuro/ Sci 2019;40:857-9.

32 Cova I, Del Tedesco F, Maggiore L, et al. Cognitive disorders in migrants: retrospective analysis in a center for cognitive disorders and dementia in Milan. Aging Clin Exp Res 2019. doi:10.1007/ s40520-019-01224-4. [Epub ahead of print: 27 May 2019]. 